\title{
EL CINE COMO METODOLOGÍA DIDÁCTICA. ANÁLISIS SISTEMÁTICO DE LA LITERATURA PARA UN APRENDIZAJE BASADO EN EL CINE (ABC)
}

\author{
Silvia díaz HeRrero* \\ Grupo Ciberimaginario (Universidad Rey Juan Carlos) \\ sdiazherrero@colegioelsalvador.com \\ Manuel Gértrudix Barrio** \\ Universidad Rey Juan Carlos \\ manuel.gertrudix@urjc.es
}

Recibido: 15/11/2020 Aceptado: 22/1/2021

doi: https://doi.org/10.26439/contratexto2021.n035.4964

RESUMEN. El uso del cine en las aulas no es una propuesta novedosa, pero en la mayoría de las ocasiones se usa como recurso didáctico y no como una metodología capaz de vehicular un aprendizaje, de forma que pueda considerarse una metodología educativa en sí misma: el aprendizaje basado en el cine ( $\mathrm{ABC}$ ). Comenzar esta transformación requiere estudiar el estado del arte para buscar las experiencias previas en las que se haya utilizado el cine en las aulas. Mediante una revisión sistemática de la literatura (artículos y libros publicados en español e inglés en los últimos veinte años) que aborda el uso del cine en el aula, se realiza una aproximación a las prácticas docentes documentadas para establecer cuáles son los rasgos distintivos que las caracterizan. No se han encontrado evidencias documentadas del uso del cine en el aula como metodología ni una correlación con la mejora en el rendimiento académico.

PALABRAS CLAVE: cine / recurso didáctico / metodología educativa / aprendizaje basado en el cine / educación

\footnotetext{
* Máster en Cine, Televisión y Medios Interactivos por la Universidad Rey Juan Carlos, España (véase: https:// orcid.org/0000-0002-5839-9910).

** Ph. D. en Ciencias de la Información por la Universidad Complutense de Madrid, España (véase: https:// orcid.org/0000-0002-5869-3116).
} 


\section{MOVIES AS AN EDUCATIONAL METHODOLOGY. A SYSTEMATIC LITERATURE ANALYSIS FOR MOVIE-BASED LEARNING (MBL)}

ABSTRACT. Using movies in the classroom is not a new proposal, but it is mostly used as an educational resource and not as a methodology capable of conducting learning so that it could be considered as an educational methodology by itself: movie-based learning (MBL). Starting this transformation requires studying the state of the art to look for previous experiences in which movies have been used in the classroom. Through a systematic literature review (articles and books published both in Spanish and English in the last twenty years) on the use of movies in the classroom, an approach to documented teaching practices was made to determine their distinctive features. Neither documented evidence on the use of movies in the classroom as a methodology nor a correlation with the improvement of students' academic performance were found.

KEYWORDS: movies / educational resource / educational methodology / moviesbased learning / education

\section{O CINEMA COMO METODOLOGIA EDUCACIONAL. REVISÃO SISTEMÁTICA DA LITERATURA PARA A APRENDIZAGEM BASEADA NO CINEMA (ABC)}

RESUMO. 0 uso do cinema na sala de aula não é uma proposta inédita, mas na maioria das vezes é utilizado como um recurso didático e não como uma metodologia capaz de transmitir uma aprendizagem e, desse modo, poder ser considerada uma metodologia educacional em si mesma: aprendizagem baseada no cinema ( $A B C)$. Iniciar essa transformação exige estudar o estado da arte na procura de experiências anteriores no uso do cinema em sala de aula. Através de uma revisão sistemática da literatura realizada sobre os artigos e livros publicados em espanhol e inglês, nos últimos vinte anos, que abordam o uso do cinema em sala de aula, é feita uma abordagem das práticas docentes documentadas para estabelecer quais são os traços distintivos que as caracterizam. Não foram encontradas evidências documentadas do uso do cinema em sala de aula como metodologia ou correlação com a melhoria do desempenho acadêmico.

PALAVRAS-CHAVE: cinema / recurso didático / metodologia educacional / aprendizagem baseada no cinema / educação 


\section{INTRODUCCIÓN}

Conseguir que los estudiantes logren un aprendizaje significativo en algunas áreas de conocimiento es una tarea complicada para los docentes, sobre todo cuando lo que se tiene que asimilar es un concepto abstracto. Para conseguir este objetivo, se propone el cine como metodología pedagógica. Como recurso, se viene utilizando desde hace muchos años y ha sido objeto de múltiples propuestas para su aplicación en las clases de todas las etapas educativas. Se pueden señalar muchas experiencias que relatan el uso del cine en diferentes áreas de conocimiento, desde las ciencias naturales hasta la historia, pasando por las matemáticas, el derecho, la medicina o la filosofía. Sin embargo, en muy pocas ocasiones, se plantea el uso del lenguaje cinematográfico para vehicular un aprendizaje. Es decir, cambiar la forma de usar el cine como un recurso didáctico para convertir el lenguaje cinematográfico en una metodología educativa.

\section{La alfabetización mediática como marco}

Desde una perspectiva histórica, debemos enmarcar el desarrollo de estas propuestas dentro de un concepto más amplio que es el de alfabetización mediática (media literacy), definido como "las competencias, los conocimientos y las capacidades de comprensión que permiten a los ciudadanos utilizar con eficacia y seguridad los medios", y que aportan "el pensamiento crítico necesario para discernir, analizar realidades complejas y reconocer la diferencia entre opiniones y hechos" (Parlamento Europeo y Consejo de la Unión Europea, 2018), así como la educomunicación (Aparici, 2011; Aguaded, Hernando Gómez y Pérez, 2012; Gálvez et al., 2017).

En ese marco, en España, ya en 1985, el Ministerio de Educación y Cultura puso en marcha el Proyecto Mercurio, que entre sus objetivos buscaba "desarrollar en profesores y alumnos la capacidad de descodificar y producir mensajes audiovisuales, aprovechando las posibilidades comunicativas y expresivas del video en el proceso de enseñanza y aprendizaje" (MEC, 1988, p. 27). En la primera década del nuevo siglo, los programas Internet en la Escuela e Internet en el Aula supusieron un nuevo impulso (Gértrudix et al., 2007), que permitió el desarrollo de contenidos educativos digitales para la enseñanza del lenguaje cinematográfico, como MEDIA Cine (CNICE, 2008) para educación secundaria y bachillerato o el proyecto Mekos para educación primaria (CNICE, 2007).

Por su parte, la Unión Europea desarrolló entre los años 2007 y 2013 los programas MEDIA y MEDIA Mundus (Comisión Europea, 2018), que implicaron un fuerte impulso para la educación en medios (Aguaded, 2013). Además, elaboró un cuerpo de recomendaciones (Comisión Europea, 2009) y financió diferentes investigaciones y grupos de trabajo dirigidos a cuestiones como el desarrollo de un marco de alfabetización mediática o el análisis del papel de la alfabetización fílmica (Directorate-General for Education, 
Youth, Sport and Culture, 2013). Recientemente ha puesto en marcha un ambicioso plan dentro del Programa Europa Creativa (Comisión Europea, 2019), que es una nueva oportunidad para el impulso de la alfabetización mediática y fílmica, y que ofrece un espacio para el planteamiento de transformar la forma de usar el cine en el aula: de ser visto como un recurso didáctico a convertirlo en una metodología educativa.

\section{Experiencias educativas en el aula}

Para iniciar esta transformación, es necesario llevar a cabo un exhaustivo análisis del estado del arte que permita documentar las experiencias previas en las que se haya usado el cine en las aulas. Así podremos hacer una aproximación al tipo de uso que los docentes realizan de este medio expresivo dentro del aula.

Las experiencias disponibles son muchas y muy variadas. En España, las encontramos en diferentes etapas educativas, como los ejemplos en secundaria, de Guerra (2004) o García (2005), pero también en la universidad, como los de García y Hernández (2013) o García (2013). Muchas de estas investigaciones toman como referencia los trabajos de Fernández Sebastián (1998), pero mucho antes Blanco (1975) reveló que la carencia de uso del cine en las aulas universitarias se debía a que en los colegios no se había introducido este recurso, y, cuando se hizo, fue de forma oficiosa y no oficial; por su parte, Ruiz-Rubio (1994) había establecido la utilidad del cine de ficción para la labor pedagógica, encontrando argumentos más que "suficientes para justificar la inclusión del cine en los proyectos curriculares". De hecho, en esta década de los noventa salen a la luz diversas experiencias referentes al uso del cine en las aulas, en las que se apunta a la necesidad de una formación adecuada del profesorado para que sea capaz de analizar el contenido audiovisual y diseñar su empleo pertinente dentro de las programaciones didácticas (Gispert, 1995).

A nivel internacional, existen muchas referencias de estas experiencias; por citar solo algunos ejemplos, podemos señalar a Mateus (2017) o a Cambra et al. (2017), así como los enfoques pedagógicos que defienden el papel de la escuela para establecer un modelo de análisis crítico-cultural que escape de las tendencias o contenidos dominantes, como la de Bergala (2007).

Se trata, en todos los casos, de aportaciones que parten, como se detalla a continuación, de perspectivas diversas, a veces complementarias y otras antagónicas, pero que valorizan la integración del cine en los procesos de aprendizaje y su impacto en la configuración, no solo de imaginarios colectivos al servicio de los sistemas de información cultural (SIC), sino como verdaderos aparatos culturales que son capaces de transferir conocimientos profundos de la realidad social (Ahumada Barajas, 2012). 


\section{Aproximaciones al uso del cine en el aula}

Las motivaciones que promueven el uso de contenido audiovisual dentro del aula también son variadas. Según algunos estudios, como el de Dark (2005), el uso de las películas fomenta el pensamiento creativo y, además, supone una ayuda visual para el aprendizaje. Es importante ahondar en este concepto porque, siguiendo las aportaciones de O'Connor y McDermott (1997), citados por Velázquez et al. (2007), el nivel de percepción de los sentidos se puede resumir en la tabla 1.

Tabla 1

Nivel de percepción de los sentidos

\begin{tabular}{lc}
\hline Técnica de contacto & Nivel de percepción \\
\hline Visual: vista & $75 \%$ \\
Auditivo: oído & $13 \%$ \\
Corporal: tacto & $6 \%$ \\
Olfativo: olfato & $4 \%$ \\
Gustativo: gusto & $2 \%$ \\
\hline
\end{tabular}

Fuente: O'Connor y McDermott (1997), citados por Velázquez et al. (2007)

Según estos autores, el $88 \%$ de nuestro nivel de percepción se concentra en lo audiovisual; de hecho, nuestra percepción es eminentemente visual, de forma que el cine se convierte en una herramienta muy potente para conseguir un aprendizaje significativo. En esta línea, Benasayag (2017) hace una reflexión muy interesante sobre las posibilidades pedagógicas de las imágenes que avalan el uso del cine en el aula; igualmente, Deleuze (1994) plantea aportes sobre el potencial que tienen las imágenes en movimiento para el pensamiento.

Cabe señalar que a partir de la adolescencia "toda la energía del alumno se orienta hacia los procesos superiores de pensamiento y aprende a aplicar a su estudio el razonamiento, el pensamiento crítico, la creatividad y la elaboración de las respuestas de forma eficaz" (Martín-Lobo y Rodríguez Fernández, 2015, p. 24), por lo que un aprendizaje basado en el cine ( $A B C$ ) puede ser una opción para aumentar el nivel de percepción y fomentar los procesos superiores de pensamiento.

Además, los procesos de identificación que llegamos a conseguir gracias al lenguaje cinematográfico, como explica González Requena (2006), pueden servir para ayudarnos a construir un aprendizaje experiencial (Kolb, 1984) que contribuya a fijar los conceptos que se estén tratando en el aula en ese momento. Esta identificación no solo nos sirve para mejorar el rendimiento académico de los estudiantes, sino que también puede ser una fuente de motivación, lo que pone de relieve el "poder de la educación mediática" (Ferrés, 2014). 
Existen propuestas en las que se usan con propósitos pedagógicos los medios audiovisuales creados por los propios estudiantes, tal como relata Leandro (2001) o como sucede en el proyecto Minuto Lumiere, que plantea una pedagogía centrada en la creación (Mastandrea y Guerra, 2019), aunque son minoritarias.

También encontramos aproximaciones, como los estudios de Calvert et al. (2007), que son contrarias a introducir medios audiovisuales en el aula, ya que se trataría de un medio pasivo. A su juicio, va en contra de las teorías de aprendizaje activo porque es un sistema de una sola vía y el estudiante no participa. Además, consideran que los estudiantes no lo perciben como un medio didáctico, sino como una forma de relajación y entretenimiento. No es un estudio aislado; podemos encontrar que algunos profesores de historia consideran que el cine es "un fantasma que amenaza los dominios de la ciencia histórica, haciendo que el gran público se conforme con historietas más o menos espectaculares, en vez de con la historia con mayúsculas" (De Pablo, 2001). Algunos autores señalan que las películas falsifican la historia y, por tanto, no creen que su uso en el aula sea beneficioso para su labor docente (Rodríguez, 2005). Otra sombra de sospecha que planea sobre el uso del cine en el aula es la motivación del profesorado para usarlo, puesto que "en casi todas las experiencias prima el uso del filme más como pretexto que como texto" (Pla Valls, 2010). Esto quiere decir que, en muchos casos, poner una película en el aula no tiene una motivación pedagógica intrínseca, sino que responde a otras casuísticas relacionadas con períodos de ampliación al final de curso, ausencia de un profesor o intervalos entre evaluaciones, es decir que muchas veces podría usarse para rellenar huecos y no como un medio de aprendizaje en sí.

Sin embargo, estos estudios obvian un componente muy importante: que el lenguaje cinematográfico y el audiovisual, por extensión, forman parte ineludible de la experiencia cotidiana de nuestros estudiantes, por lo que la exposición a las múltiples pantallas construye, inevitablemente, aprendizajes no formales que tienen un extraordinario peso en su forma de percibir y relacionarse con la realidad. El período de confinamiento al que la sociedad española se ha visto sometida en el 2020 ha amplificado esta exposición a los relatos audiovisuales. Según los paneles de CNMC, durante este tiempo, el consumo de contenidos en las plataformas $\mathrm{OTT}^{1}$ a través de sistemas $\mathrm{VoD}^{2}$ ha aumentado significativamente. Así, las suscripciones a estos servicios, que en el 2015 eran algo anecdótico y llegaron al $38 \%$ en el 2019, han experimentado un crecimiento exponencial. Según un estudio de Reelgood (Santos, 2020), desde el 2 de marzo al 13 de abril del 2020 ha aumentado la actividad en las plataformas $\mathrm{SVoD}^{3}$ un $157 \%$, como se puede observar en la figura 1.

1 Over the top, que ofrece contenidos de video a través de internet.

2 Video on demand, que ofrece contenidos bajo demanda.

3 Subscription video on demand, que ofrece suscripción a un catálogo que se puede consumir bajo demanda. 


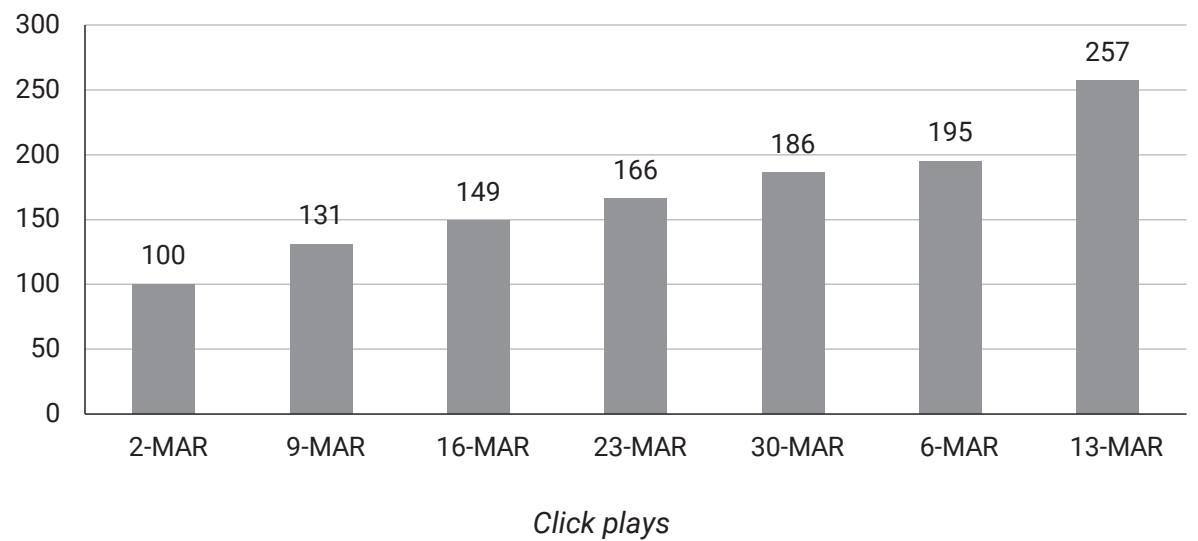

Figura 1. Aumento del uso de las plataformas SVoD

Elaboración propia a partir de los datos de Reelgood (Santos, 2020)

A esto se debe sumar el aumento del consumo de minutos de video en un $46,59 \%$, según la encuesta de hábitos y prácticas culturales en España de $\operatorname{MCUD}(2015,2019)$, en detrimento de los minutos de televisión, cuya tendencia no solo ha continuado en ascenso, sino que ha sufrido un incremento muy pronunciado, como revela el estudio de Barlovento Comunicación (2020). En él se puede confirmar cómo los minutos en los que se usa la televisión para otros contenidos no propiamente televisivos crecieron significativamente en el mes de marzo y se dispararon durante el mes de abril del 2020.

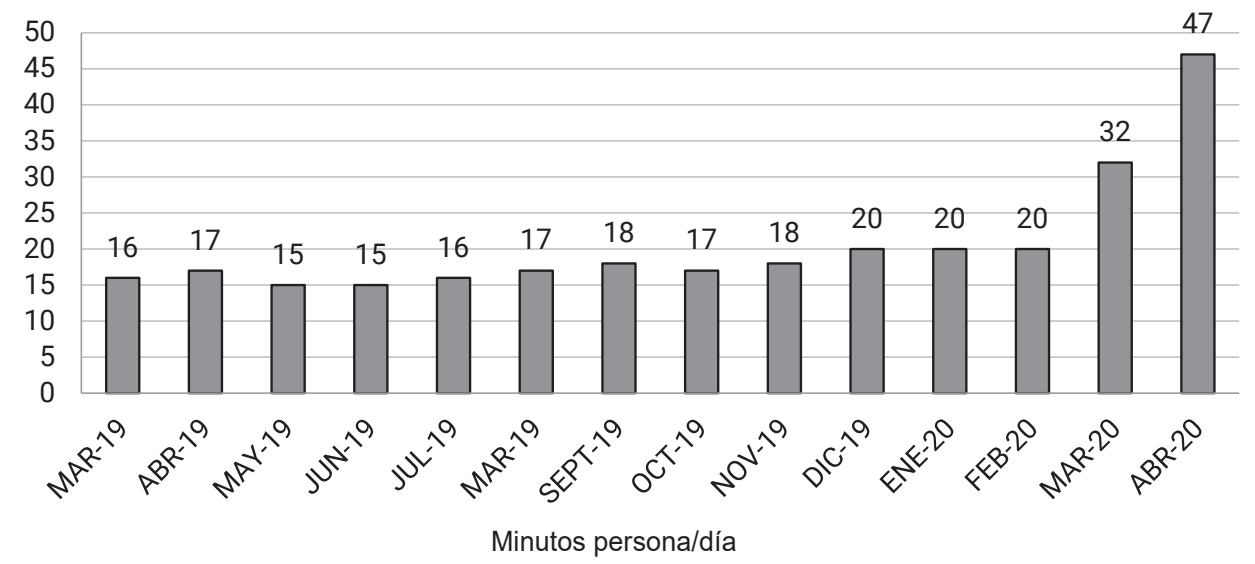

Figura 2. Minutos de televisión consumidos para otros usos

Elaboración propia a partir de los datos de Barlovento Comunicación (2020) 
Con estos datos, podemos confirmar que el lenguaje cinematográfico sobre el que se construyen los productos audiovisuales no solo es una forma más de comunicación para nuestro público objetivo, los estudiantes, sino que es la principal. El lenguaje audiovisual les es cercano y cotidiano, por lo que se convierte en un medio muy eficaz para aproximarnos a nuestro alumnado, aumentar su motivación y mejorar el rendimiento académico.

\section{METODOLOGÍA}

\section{Objeto}

El objeto de estudio es el cine desde su dimensión educacional, con la finalidad de examinar si los estudios publicados en la literatura científica muestran que es utilizado como metodología en las aulas de distintas etapas educativas y cuáles son los resultados de dicho uso.

\section{Objetivos, preguntas de investigación y técnicas de investigación}

Se ha llevado a cabo una revisión sistemática de la literatura (SLR) con el objetivo de identificar los estudios sobre el uso del cine como metodología docente. La SLR es un recurso que sirve para identificar, comprender y evaluar la información disponible sobre un campo de investigación durante un período de tiempo. Atendiendo a la metodología establecida por Higgins y Green (2006), Kitchenham (2004) y el Centre for Reviews and Dissemination de York University (2009), el estudio se ha desarrollado en tres fases:

\section{Fase 1. Planificación}

En la primera fase, se definió la estrategia de búsqueda en las bases de datos tomando las decisiones pertinentes para orientarla. La búsqueda se ha realizado a partir de un conjunto de preguntas que sirven para la elaboración del protocolo para la revisión:

- ¿Cuántos estudios hay sobre la aplicación del cine como metodología educativa?

- ¿En qué etapas educativas se ha puesto en práctica esta metodología educativa?

- ¿Cómo ha evolucionado el rendimiento académico de los estudiantes cuando se ha puesto en práctica?

La selección de los estudios para revisar se ha guiado por los siguientes criterios:

- Bases de datos: inicialmente se seleccionaron WoS y Scopus. Debido a que el número de referencias recuperadas fue limitado, se amplió la búsqueda en las bases de datos Crossref y Google Scholar. Según el estudio de MartínMartín et al. (2021), la superposición de los resultados de estas bases de datos 
garantizaría una aproximación adecuada a la muestra de análisis.

- Palabras clave: los términos seleccionados han sido cine (cinema, ciné) y metodología (metodology, méthodologie).

También se han establecido algunos criterios de exclusión:

- Ventana temporal: desde el 2000 hasta el 2019.

- Idioma: español, inglés y francés. Durante la extracción de resultados, aquellos que se han encontrado en portugués se han incorporado al análisis.

- Tipo de documento: se han eliminado las referencias que no corresponden al campo de la educación, al igual que las que no tratan de forma explícita el cine, las relacionadas con educación no formal, las citas y reseñas de otros estudios, y la literatura gris.

\section{Fase 2. Conducción}

Para llevar a cabo esta fase, se utilizó la herramienta Publish of Perish. Aplicando los criterios de exclusión y siguiendo las decisiones que han pautado la búsqueda, se llevaron a cabo 12 búsquedas (las dos palabras clave en los tres idiomas elegidos y en las cuatro bases de datos seleccionadas). Luego se realizó el filtrado para eliminar los duplicados y los tipos de documentos que respondían a los criterios de exclusión. En esta fase se ha intentado dar respuesta a la primera pregunta que ha orientado la revisión, referida a cuántos estudios hay sobre la aplicación del cine como metodología educativa. El listado final ofreció un total de 161 referencias, que constituyen la muestra de análisis.

\section{Fase 3. Informe de resultados}

Para proceder con el análisis, se elaboró un formulario (ver el anexo), adaptado del Cuestionario para el Análisis Sistemático de Literatura (Modelo SLR Ciberimaginario) (Gértrudix et al., 2018), para procesar y sistematizar la información, que posteriormente se analizará para extraer conclusiones. Las respuestas a ese formulario se han ajustado a un vocabulario controlado (lista normalizada de temas y de identificadores) propuesto por UNAM, IISUE (2017). En el formulario se han recogido los datos para intentar dar respuesta a las otras dos preguntas que han orientado la revisión: ¿en qué etapas educativas se ha puesto en práctica esta metodología educativa? ¿Cómo ha evolucionado el rendimiento académico de los estudiantes cuando se ha puesto en práctica? 


\section{RESULTADOS}

Al fijar las decisiones para pautar la búsqueda, se puso de manifiesto que no sería posible usar solamente Scopus y WoS como fuentes de información, ya que el número de referencias que se encontraron eran muy limitadas, por lo que se decidió incluir también los resultados de otras bases de datos: Google Scholar y Crossref. De esta forma, la cantidad de estudios por analizar aumentó considerablemente. De hecho, en el siguiente gráfico se puede observar que la inmensa mayoría de las referencias seleccionadas proceden de Google Scholar.

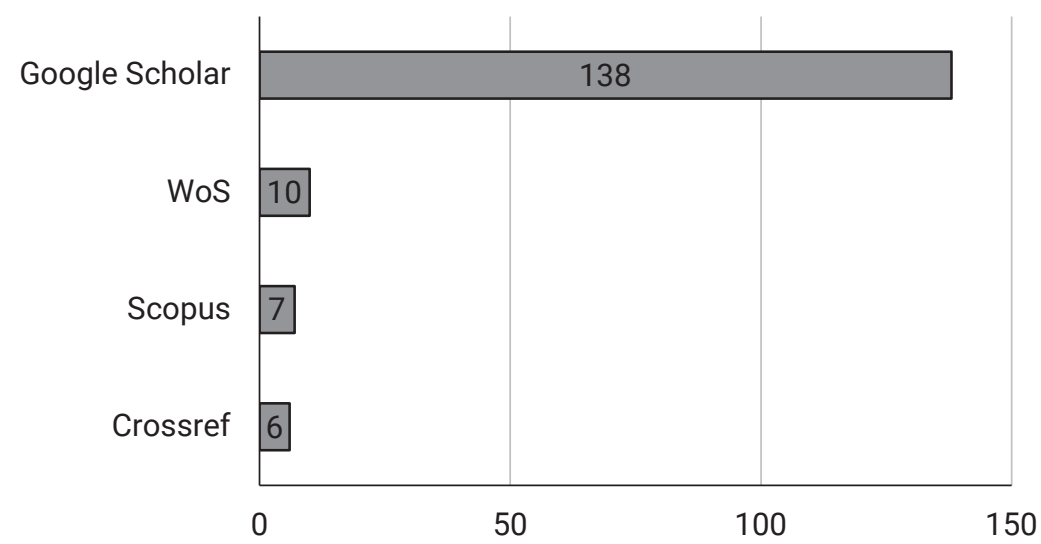

Figura 3. Distribución de referencias por base de datos

\section{Elaboración propia}

Aun ampliando la búsqueda en más bases de datos, la mayoría de los resultados obtenidos han sido en español. En los demás idiomas, el número de resultados ha sido menor, por lo que es posible que la traducción literal de los términos no sea la más adecuada en este contexto y haya que realizar la búsqueda recurriendo a otras palabras clave que puedan utilizarse en estudios de referencia internacional en este ámbito, especialmente en la literatura anglosajona.

El $72,67 \%$ de los estudios analizados corresponden a los 10 últimos años. Esto demuestra que hay una clara tendencia en el interés de los docentes por introducir el cine en las aulas, especialmente en la enseñanza universitaria. Tal como se puede observar en la figura 4, el número de estudios llevados a cabo en la enseñanza universitaria es muy superior al del resto de las etapas educativas, siendo educación infantil y primaria las que presentan menos referencias. La explicación a este hecho puede ser la escasa motivación que tienen los docentes de enseñanza no universitaria para publicar o hacer investigaciones y publicaciones académicas; la universidad, su estructura y recursos, es el lugar donde se realizan la mayoría de estas. 


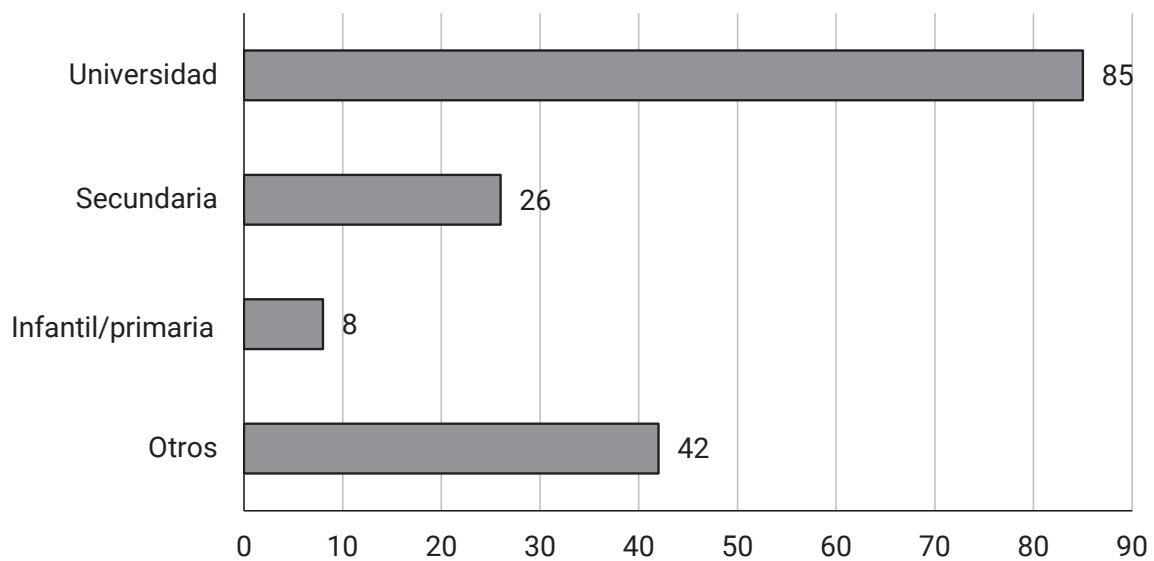

Figura 4. Distribución de referencias por etapa educativa

\section{Elaboración propia}

Si analizamos el número de citas, los resultados arrojan datos muy interesantes, que se pueden observar en la figura 5 . El 72,67 \% de las referencias tienen menos de 10 citas, lo que nos indica que son artículos con muy poco impacto.

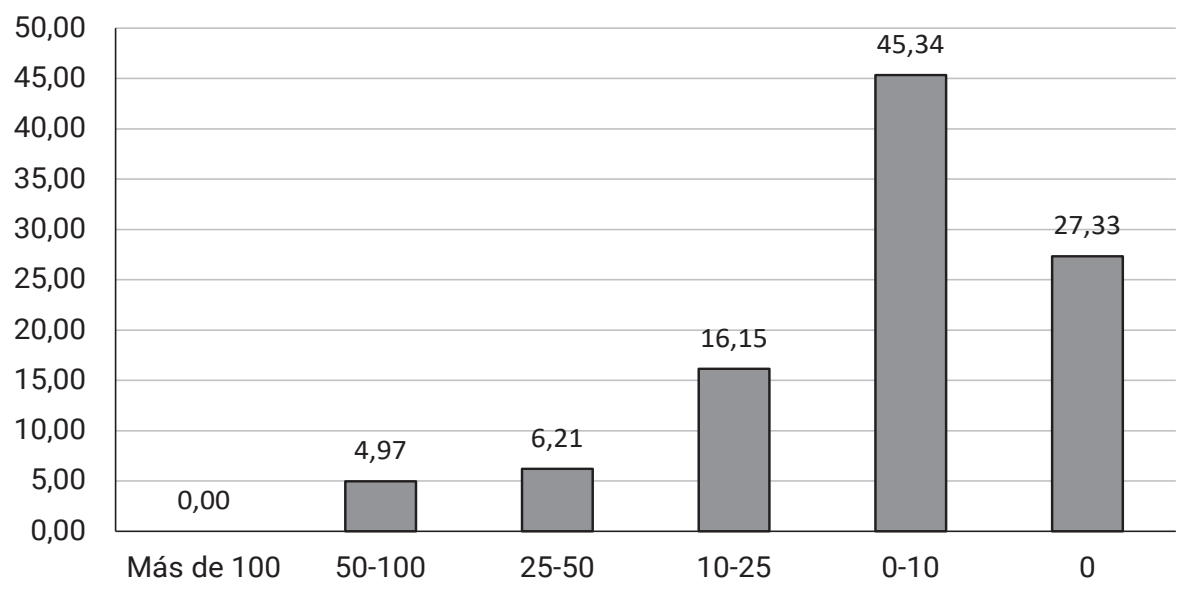

Figura 5. Número de citas por referencia

\section{Elaboración propia}

El escaso impacto de estos estudios puede estar relacionado con el tipo de investigación que han llevado a cabo. En el formulario de recojo de información, la variable tipo de investigación tomaba los siguientes valores: descriptiva, comparativa, analítica, 
explicativa y participativa. Solo el $15,53 \%$ de los estudios han llevado a cabo uno de esos tipos de investigación. El 55,28 \% expone propuestas de uso en el aula y el $18 \%$ plantea el uso del cine como recurso didáctico. Sin embargo, no hay apenas investigaciones que demuestren la relación entre el uso del cine y otras variables como el rendimiento académico o la adquisición de conceptos abstractos, sino que se limitan a exponer la experiencia y a valorarla a través de cuestionarios de opinión que contestan los estudiantes tras haber llevado a cabo la actividad propuesta. Para añadir el tipo de investigación al que se refiere cada artículo, se ha utilizado el vocabulario controlado propuesto por UNAM, IISUE (2017).

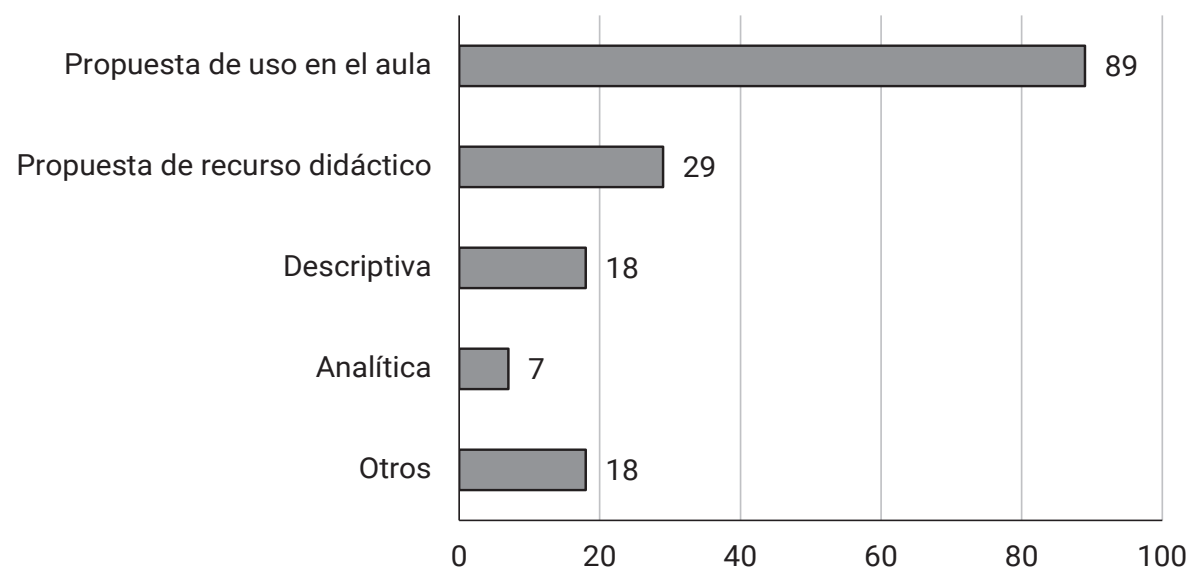

Figura 6. Distribución de referencias por tipo de investigación

\section{Elaboración propia}

Esto nos lleva al tipo de uso que se hace de los contenidos audiovisuales en las aulas. Se puede observar en la figura 7 que en el $88 \%$ de los estudios analizados el cine se ha empleado como recurso. Solamente en un $7 \%$ de ellos se plantea como metodología de aprendizaje. Es decir, el uso del cine en las aulas se propone como una actividad puntual o como un complemento, pero no como una metodología educativa que sirva para vehicular el aprendizaje. 


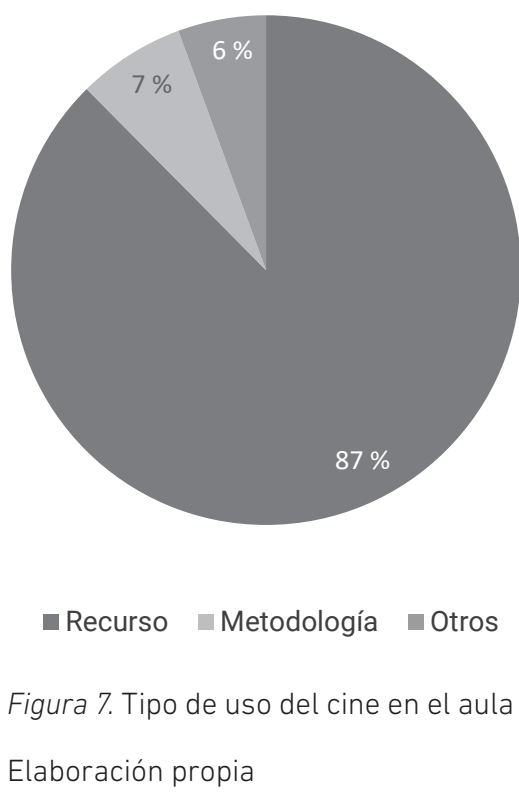

Hay una amplia diversidad en los abordajes disciplinares desde los que se realizan estos estudios. Así, encontramos propuestas desde la perspectiva del derecho, de la medicina, la historia, la microbiología y la investigación, y no faltan estudios que lo que proponen es un acercamiento interdisciplinar de las bondades de este recurso, lo que nos lleva a reflexionar sobre la transversalidad que nos ofrece el cine en el aula.

La mayoría de estas propuestas hablan de experiencias concretas que describen una actividad que se ha puesto en práctica por parte del profesorado. Se estudia y evalúa la experiencia específica, pero no es extrapolable a otras circunstancias ni contextos. Y a pesar de que encontramos movimientos como el que promueve la revista Medicina y Cine de la Universidad de Salamanca para la divulgación del uso del cine con fines educativos, estos trabajos no llevan a cabo estudios experimentales en los que se ahonde en la comparación o en la correlación para justificar la elección de este recurso.

También se encuentran muchas referencias que son programaciones didácticas de una asignatura concreta, mientras que otras hablan de las bondades del cine y su aplicación pedagógica sin especificar el ámbito de conocimiento ni la etapa educativa en la que se desarrollan. Por tanto, se hace muy complicado poder tomar estas propuestas como punto de partida para promover una metodología educativa basada en el cine.

Estas características muestran que el tipo de documento más analizado no ha tenido la forma de artículo académico o de investigación al uso, a pesar de ser documentos oficiales (programaciones didácticas, por ejemplo). 


\section{DISCUSIÓN}

El análisis de los resultados permite extraer las siguientes conclusiones. La primera es que la mayoría de los estudios analizados versan sobre aplicaciones docentes del cine dentro del aula, pero no encontramos un análisis posterior de esas experiencias, a lo sumo, encuestas de opinión de los propios estudiantes. Se observa una falta de investigación sistemática y rigurosa que incida en la relación del medio audiovisual con un aprendizaje significativo. Estos estudios se basan, en su mayor parte, en dar cuenta de una experiencia concreta y el análisis, cuando lo hay, suele estar centrado en encuestas de opinión para que los estudiantes valoren el recurso, pero no ofrecen información sobre si hay un aumento en su rendimiento académico. Por ello, se vuelve necesario un mayor número de investigaciones que incluyan esos ítems para poder valorar su utilidad y eficiencia.

Este limitado alcance puede ser la causa de que sean artículos con poco impacto; es decir, se citan poco y, por tanto, no llegan ni sirven de referencia para la comunidad docente, lo que provoca que muchas de esas propuestas sean similares y no se mejoren o se amplíen con el tiempo. Tampoco se toman como punto de partida para investigaciones futuras.

La mayoría de las referencias analizadas dan cuenta de experiencias llevadas a cabo en el ámbito universitario. Este hecho puede deberse a que las experiencias en otras etapas educativas no se publican, debido a que la formación de esos docentes no incluye tareas de investigación, o bien porque los incentivos son menores o no tienen la estructura necesaria para hacerlo. Por ello, otra propuesta de investigación podría ser cuán extendido está el uso del cine en las aulas, pero tomando muestras significativas en todas las etapas educativas, no solo en la universitaria. De esa forma se podrá extraer información de la presencia real de este recurso en la educación. Lo que se ha explorado en esta revisión de la literatura no se corresponde con la expansión del recurso entre la comunidad docente, porque hay etapas educativas que están subrepresentadas y, además, no se puede saber con exactitud cuán extendido se encuentra el uso del cine en las aulas.

Ninguna de estas investigaciones despeja la duda sobre si se trata de un medio que genera distracción en los estudiantes, tal como apuntaban Calvert et al. (2007). En todas ellas conocemos la opinión de los estudiantes que han realizado la actividad, pero no la percepción que tiene el profesorado sobre el uso del cine en el aula. Podría ser que coincidan también en verlo como una distracción y, por ello, no lo utilicen de una forma sistemática y recogida en sus programaciones docentes. De hecho, el análisis que se lleva a cabo, en la mayoría de las referencias analizadas que lo contienen, se basa en encuestas de opinión en las que se hace hincapié en si los estudiantes consideran que ha sido un recurso eficaz para alcanzar los objetivos propuestos y si creen que les ha 
ayudado a consolidar conocimientos. Esta preocupación por la opinión del alumnado, y que sean ellos mismos los que confirmen su utilidad, puede responder al calado que tienen algunas de las opiniones que se pueden leer en De Pablo (2001). Sin embargo, al no realizar un estudio experimental en el que se confirme el efecto del cine sobre el rendimiento académico o el hecho de que se referencien de forma mayoritaria únicamente actividades puntuales confirma lo que ya desvelaba Pla Valls (2010) en relación con el uso del cine como recurso, porque la mayoría de los docentes lo emplea en actividades cuyo peso en la ponderación en el total de la evaluación es muy escasa.

Parece que rellenar huecos o tratar contenidos transversales que se escapan del temario principal de la asignatura sigue siendo el uso mayoritario del cine entre los docentes, aspecto que ya trataban de superar los programas institucionales de mediados de los años ochenta (MEC, 1988). Aunque esto es algo que se podría confirmar mediante una futura investigación en la que se analicen las prácticas docentes y la percepción del uso del cine en las aulas por parte del profesorado, y que describa, además, el uso que hacen estos docentes del medio audiovisual, así como la percepción y valoración sobre la utilización de este recurso por el resto del profesorado. En este estudio se ha demostrado que la mayor parte de su uso está relacionado con actividades puntuales o complementarias y en muy pocas ocasiones se ha utilizado como metodología docente, lo que confirma que es una línea de investigación que se debe explorar.

A pesar de que el $72,67 \%$ de los estudios corresponden a los 10 últimos años, esto no demuestra que su uso sea continuado en el tiempo y esté consolidado, pues podría tratarse de actividades puntuales de innovación. Es cierto que se observa una tendencia ascendente en la introducción del cine en las aulas, pero no sabemos la continuidad de esas actividades ni sus posibilidades de extenderse en otros ámbitos.

Estas conclusiones evidencian la falta de experiencia metodológica sobre el uso del cine en las aulas. Es decir, el aprendizaje basado en el cine $(\mathrm{ABC})$ no está documentado como metodología, lo que responde a una de las cuestiones que motivan este estudio, y, por tanto, se abre una línea de investigación en la que se intentará demostrar que es un elemento capaz de conseguir que los estudiantes aumenten su rendimiento académico, así como facilitarles la adquisición de conceptos abstractos. Así, se concluye que el cine no es solo un recurso didáctico, sino que puede ser una metodología de aprendizaje que es posible sistematizar y extrapolar a todos los ámbitos docentes.

Para proponer la aplicación de esta metodología se requiere comprobar, en primer lugar, las experiencias previas del uso del cine en las aulas, para lo cual se ha llevado a cabo el presente estudio.

Las conclusiones nos permiten comprobar que, a pesar de que el cine está presente en las aulas de todas las etapas educativas, no se usa como metodología de aprendizaje, sino únicamente como recurso. Esto nos abre nuevas vías de trabajo orientadas al diseño 
y validación de un método específico que desarrolle el $A B C$ (aprendizaje basado en el cine) como metodología didáctica que facilite el aprendizaje de conceptos abstractos que son nuevos para el alumnado.

Esta línea de investigación, que contempla la evaluación de un protocolo de intervención en el aula, así como la de los instrumentos de recogida de información, nos llevará a establecer si los estudiantes son capaces de analizar, entender y producir información relacionada con los conceptos abstractos que se hayan programado en los objetivos. Mediante la validación del método por expertos, se procederá a realizar un modelo con un diseño experimental, gracias al cual se pueda comprobar si existe correlación entre la metodología y el aprendizaje significativo, recabando información sobre el nivel de adquisición de los conceptos a través de los instrumentos de recogida que hayan sido verificados.

\section{REFERENCIAS}

Aguaded, J. I. (2013). El Programa "Media" de la Comisión Europea, apoyo internacional a la educación en medios. Comunicar: Revista Científica de Comunicación y Educación, (40), 7-8.

Aguaded, J. I., Hernando Gómez, Á., y Pérez, M. A. (2012). Pantallas en la sociedad audiovisual: edu-comunicación y nuevas competencias. Comunicação e Sociedade, 21, 217-230.

Ahumada Barajas, R. (2012). El nuevo escenario mediático y la reformulación de los estudios de recepción. Quórum Académico, 9(1), 11-29.

Aparici, R. (2011). Educomunicación: más allá del 2.0. Gedisa.

Barlovento Comunicación. (16 de abril del 2020). Análisis. Otros usos y consumos del televisor. Abril 2020. https://www.barloventocomunicacion.es/informes-barlovento/ analisis-otros-usos-y-consumos-del-televisor-abril-2020

Benasayag, A. (2017). La hipótesis de la cinefilia docente. El cine de ficción en la escuela media argentina. Revista Digital do LAV, 10(2), 56-78. doi: 10.5902/1983734827504

Bergala, A. (2007). La hipótesis del cine. Pequeño tratado sobre la transmisión del cine en la escuela y fuera de ella. Laertes.

Blanco, J. L. (1975). El cine en las aulas. Padres y Maestros / Journal of Parents and Teachers, (48), 22-26.

Calvert, B., Casey, B., Casey, N., French, L., y Lewis, J. (2007). Television studies: the key concepts. Routledge. 
Cambra, I., Mastandrea, P., Paragis, M. P., Tomas, A., González, F., y Michel, J. J. (2017). Cine aplicado a la psicología: el abordaje ético-clínico de las tecnologías de reproducción humana asistida. Premio Facultad de Psicología, 11-39.

Centre for Reviews and Dissemination (Ed.). (2009). Systematic reviews. CRD's guidance for undertaking reviews in health care. CRD, University of York.

CNICE (MEC). (2007). Proyecto Mekos: integración curricular de los medios de comunicación social en educación primaria. http://ares.cnice.mec.es/mcs/

CNICE (MEC). (2008). MEDIA Cine. http://recursos.cnice.mec.es/media/cine/

Comisión Nacional de los Mercados y la Competencia (CNMC). (2019). Panel de hogares del tercer trimestre de 2019. http://data.cnmc.es/datagraph/

Comisión Europea. (2009). Commission Recommendation of 20 August 2009 on media literacy in the digital environment for a more competitive audiovisual and content industry and an inclusive knowledge society. http://data.europa.eu/eli/reco/2009/625/oj

Comisión Europea. (23 de abril del 2018). Programa MEDIA (2007-2013). https://ec.europa. eu/programmes/creative-europe/previous-programmes/media_es

Comisión Europea. (1 de junio del 2019). Europa Creativa. https://ec.europa.eu/program mes/creative-europe/index_es

Dark, M. L. (2005). Using science fiction movies in introductory Physics. The Physics Teacher, 43, 463-465.

De Pablo, S. (2001). Cine e historia: ¿la gran ilusión o la amenaza fantasma? Historia Contemporánea, 22, 9-28.

Deleuze, G. (1994). La imagen-movimiento. Estudios sobre cine 1. Paidós.

Directorate-GeneralforEducation, Youth, Sportand Culture(EuropeanCommission). (2011). Testingandrefining,criteriatoassessmedialiteracylevelsinEurope:finalreport.https:// op.europa.eu/en/publication-detail/-/publication/4cbb53b5-689c-4996-b36b -e920df63cd40/language-en

Directorate-General for Education, Youth, Sport and Culture (European Commission). (2013). Screening literacy: executive summary. https://op.europa.eu/en/publication -detail/-/publication/cbc5f0fb-3a04-4b44-898b-8b61b0b1c520/language-en

Fernández Sebastián, J. (1998). Cine e historia en el aula. Akal.

Ferrés, J. (2014). Las pantallas y el cerebro emocional. Gedisa.

Gallardo-Camacho, J. (13 de mayo del 2020). Las nuevas audiencias en tiempos de coronavirus. The Conversation. https://theconversation.com/las-nuevas-audien cias-en-tiempos-de-coronavirus-137347 
Gálvez, M. d. C., Gértrudix, M., y García, F. (2017). Educomunicación: un marco teórico para la alfabetización mediática en la enseñanza. En E. Said Hung y F. J. Durán Ruiz (Eds.), Educación, participación y escenarios digitales: debates sobre la mediación digital en el siglo XXI (pp. 71-77). Comares.

García, F. (2005). Star Trek: un viaje a las leyes de la dinámica. Revista Eureka sobre Enseñanza y Divulgación de las Ciencias, 2(1), 79-90.

García, L., y Hernández, P. (2013). El uso del cine en la enseñanza de la psiquiatría: una propuesta metodológica. Ética y Cine Journal, 3(3), 55-61.

García, S. (2013). La materia Cine y publicidad en los estudios sobre Publicidad y Relaciones Públicas. Propuesta de planificación y metodología docente. Historia y Comunicación Social, 18(Esp.), 119-131.

Gértrudix, M., Álvarez, S., Galisteo, A., Gálvez, M. d. C., y Gértrudix, F. (2007). Acciones de diseño y desarrollo de objetos educativos digitales: programas institucionales. RUSC. Universities and Knowledge Society Journal, 4(1), 14-25.

Gértrudix, M., Rajas-Fernández, M., y Gértrudix, F. (2018). Cuestionario para el Análisis Sistemático de Literatura (Modelo SLR Ciberimaginario) (Versión 1). Zenodo. doi: $10.5281 /$ zenodo.3663317

Gispert, E. (1995). El cine como objeto y materia de estudio: la experiencia de Drag Màgic. Comunicar, 5, 69-72.

González Requena, J. (2006). Clásico, manierista, postclásico. Los modos del relato en el cine de Hollywood. Castilla Ediciones.

Guerra, C. (2004). Laboratorios y batas blancas en el cine. Revista Eureka sobre Enseñanza y Divulgación de las Ciencias, 1(1), 52-63.

Higgins, J., y Green, S. (Eds.). (2006). Cochrane handbook for systematic reviews of interventions 4.2.6. https://training.cochrane.org/handbook/archive/v4.2.6

Kitchenham, B. (2004). Procedures for performing systematic reviews. Keele University: Technical Report TR/SE-0401 and NICTA Technical Report 0400011T.1. https://goo. $\mathrm{gl} / \mathrm{wQcMaS}$

Kolb, D. (1984). Experiential learning: experience as the source of learning and development. Prentice Hall.

Leandro, A. (2001). Da imagem pedagógica à pedagogia da imagem. Comunicação \& Educação, (21), 29-36.

Martín-Lobo, P., y Rodríguez Fernández, A. (2015). La intervención desde la base neuropsicológica y metodologías que favorecen el rendimiento escolar. En P. 
Martín-Lobo (Coord.), Procesos y programas de neuropsicología educativa (pp. 14-33). Secretaría General Técnica, Centro de Publicaciones; Ministerio de Educación, Cultura y Deporte.

Martín-Martín, A., Thelwall, M., Orduna-Malea, E., y Delgado, E. (2021). Google Scholar, Microsoft Academic, Scopus, Dimensions, Web of Science, and OpenCitations' $\mathrm{COCl}$ : a multidisciplinary comparison of coverage via citations. Scientometrics, 126(1), 871-906. https://arxiv.org/abs/2004.14329

Mastandrea, P., y Guerra, N. (2019). El Minuto Lumiere como modo de crear en el aula universitaria. Comunicación presentada en el XI Congreso Internacional de Investigación y Práctica Profesional en Psicología. XXVI Jornadas de Investigación. XV Encuentro de Investigadores en Psicología del MERCOSUR. I Encuentro de Investigación de Terapia Ocupacional. I Encuentro de Musicoterapia. Facultad de Psicología, Universidad de Buenos Aires.

Mateus, J. C. (2017). Las teleseries también educan. Una defensa de las ficciones televisivas como dispositivos de aprendizaje. En G. Cappello (Ed.), Ficciones cercanas. Televisión, narración y espíritu de los tiempos (pp. 179-195). Universidad de Lima, Fondo Editorial.

Ministerio de Cultura y Deporte (MCUD). (2015). Encuesta de hábitos y prácticas culturales en España. https://www.culturaydeporte.gob.es/servicios-al-ciudadano/estadis ticas/cultura/mc/ehc/2014-2015/presentacion.html

Ministerio de Cultura y Deporte (MCUD). (2019). Encuesta de hábitos y prácticas culturales en España. https://www.culturaydeporte.gob.es/servicios-al-ciudadano/estadis ticas/cultura/mc/ehc/2018-2019/presentacion.html

Ministerio de Educación y Cultura de España (MEC). (1988). Proyectos Atenea y Mercurio. Programa de Nuevas Tecnologías de la Información y de la Comunicación (P. N. T. I. C). Secretaría de Estado de Educación. https://sede.educacion.gob.es/ publiventa/d/21621/19/0

O'Connor, J., y McDermott, I. (1997). Lenguaje corporal. Plaza \& Janés.

Parlamento Europeo y Consejo de la Unión Europea. (14 de noviembre del 2018). Directiva (UE) 2018/1808 del Parlamento Europeo y del Consejo de 14 de noviembre de 2018 por la que se modifica la Directiva 2010/13/UE sobre la coordinación de determinadas disposiciones legales, reglamentarias y administrativas de los Estados miembros relativas a la prestación de servicios de comunicación audiovisual (Directiva de servicios de comunicación audiovisual), habida cuenta de la evolución de las realidades del mercado. https://www.boe.es/doue/2018/303/ L00069-00092.pdf 
Pla Valls, E. (2010). Historia en el cine, cine en la historia. https://www.cinehistoria.com/ historia_en_el_cine.pdf

Rodríguez, F. (2005). El cine cambia la historia. Rialp.

Ruiz-Rubio, F. (1994). Cine y enseñanza. Comunicar: Revista Científica de Comunicación y Educación, (3), 74-80. doi: 10.3916/C03-1994-10

Santos, M. (8 de mayo del 2020). SVOD and AVOD streaming service performance during social distancing [mensaje en un blog]. https://blog.reelgood.com/svod-andavod-streaming-service-performance-during-social-distancing

UNAM, IISUE. (2017). Vocabulario controlado. Perspectivas Docentes, 28(63). http:// revistas.ujat.mx/index.php/perspectivas/article/view/2647

Velázquez, S., Díaz, J. J., y Velásquez, R. (2007). La ergonomía cognitiva; accesando a la percepción visual-auditivo-corporal. Ergonomía Ocupacional: Investigaciones y Aplicaciones, 1, 218-238. 


\section{ANEXo}

\section{FORMULARIO DE ANÁLISIS}

\section{Sección 1: Diseño}

1. Referencia:

2. DOI/URL:

3. Año:

4. Número de citas:

5. Base de datos:
a. Crossref
b. Google Scholar
c. WoS
d. Scopus
e. Otra

6. Idioma:
a. Español
b. Inglés
c. Francés
d. Portugués
e. Otro

7. Objeto de estudio nivel 1 :

8. Objeto de estudio nivel 2: Uso
a. Recurso
b. Metodología
c. Otro

9. Objeto de estudio nivel 2: Etapa educativa
a. Infantil/primaria
b. Secundaria
c. Universidad
d. Otro 
10. Objeto de estudio nivel 2: Disciplina

11. ¿Qué tipo de investigación es?

a. Descriptiva

i. Categorizar

ii. Caracterizar

iii. Clasificar

iv. Definir

v. Describir

vi. Detallar

vii. Detectar

viii. Diagnosticar

ix. Estudiar

x. Identificar

xi. Enumerar

xii. Especificar

xiii. Otro

b. Comparativa

i. Asociar

ii. Asemejar

iii. Comparar

iv. Contrastar

v. Determinar

vi. Diferenciar

vii. Otro

c. Analítica
i. Analizar
ii. Criticar
iii. Descomponer
iv. Establecer
v. Otro 
d. Explicativa

i. Analizar

ii. Criticar

iii. Descomponer

iv. Establecer

v. Otro

e. Participativa

i. Actuar

ii. Intervenir

iii. Modificar

iv. Participar

v. Otro

12. ¿Cuáles son las hipótesis?

13. ¿Cuál es el universo de estudio?

14. ¿Qué tipo/técnicas/métodos de muestreo utiliza?
a. Estructural
b. Intencionales o de conveniencia
c. Probabilístico
d. Significativas de la población
e. Otro

15. ¿Cuál es el tamaño de la muestra?

\section{Sección 2: Recogida de información}

1. ¿Cuántos tipos de técnicas de recogida de información hay?
a. 1
b. 2 o más

2. ¿Cuál es la técnica de recogida de información dominante?
a. Entrevistas
b. Encuestas
c. Experimentos 

d. Documentos
e. Observaciones
f. Conversaciones
g. Otra

3. ¿Cuál es la técnica de recogida de información principal? Entrevistas
a. Delphi
b. Grupos de discusión
c. Entrevistas individuales
d. Entrevista focalizada
e. No consta
f. Otra

4. ¿Cuál es la técnica de recogida de información principal? Encuestas
a. Encuesta de actitud
b. Encuesta de opinión
c. No consta
d. Otra

5. ¿Cuál es la técnica de recogida de información principal? Experimentos
a. Experimentos de campo
b. Experimentos de grupo
c. Experimentos de sujeto
d. No consta
e. Otra

6. ¿Cuál es la técnica de recogida de información principal? Documentos
a. Análisis de contenido
b. Análisis de discurso
c. Análisis documental
d. No consta
e. Otra

7. ¿Cuál es la técnica de recogida de información principal? Observaciones
a. Autoobservación 

b. Observación sistemática
c. Observación participante
d. No consta
e. Otra

8. ¿Cuál es la técnica de recogida de información secundaria?
a. Entrevistas
b. Encuestas
c. Experimentos
d. Documentos
e. Observaciones
f. Conversaciones
g. Otra

9. ¿Cuál es la técnica de recogida de información secundaria? Entrevistas
a. Delphi
b. Grupos de discusión
c. Entrevistas individuales
d. Entrevista focalizada
e. No consta
f. Otra

10. ¿Cuál es la técnica de recogida de información secundaria? Encuesta
a. Encuesta de actitud
b. Encuesta de opinión
c. No consta
d. Otra

11. ¿Cuál es la técnica de recogida de información secundaria? Experimentos
a. Experimentos de campo
b. Experimentos de grupo
c. Experimentos de sujeto
d. No consta
e. Otra 
12. ¿Cuál es la técnica de recogida de información secundaria? Documentos
a. Análisis de contenido
b. Análisis de discurso
c. Análisis documental
d. No consta
e. Otra

13. ¿Cuál es la técnica de recogida de información secundaria? Observaciones
a. Autoobservación
b. Observación sistemática
c. Observación participante
d. No consta
e. Otra

14. ¿Cómo son los datos obtenidos?
a. Datos primarios numéricos
b. Datos primarios no numéricos
c. Datos secundarios numéricos
d. Datos secundarios no numéricos

15. ¿Cuál es la naturaleza de los datos?
a. Datos cuantitativos continuos
b. Datos cuantitativos discretos
c. Datos cualitativos binarios
d. Datos cualitativos categóricos

16. ¿Cómo se han obtenido esos datos?
a. Datos declarados
b. Datos observados
c. Datos experimentales
d. Modelos o simulaciones
e. Otro 


\section{Sección 3: Análisis}

1. ¿Qué teorías se referencian?

2. ¿Cuántos tipos de técnicas de análisis se utilizan?
a. 1
b. 2 o más

3. ¿Cuál es el tipo de análisis principal que se realiza?
a. Cualitativo
b. Cuantitativo
c. No consta

4. ¿Qué tipo de análisis estadístico o matemático se realiza en función del número de variables?
a. Univariable
b. Bivariable
c. Multivariable confirmatorio
d. Multivariable exploratorio

5. ¿Qué tipo de análisis cualitativo se realiza?
a. Inducción analítica
b. Teoría fundamentada
c. Semiótico-estructural

6. ¿Qué tipo de análisis univariable se realiza?

7. ¿Qué tipo de análisis bivariable se realiza?

8. ¿Qué tipo de análisis multivariable confirmatorio se realiza?
a. Componentes principales
b. Regresión múltiple
c. Correlaciones canónicas
d. Discriminante
e. Factorial
f. Varianza
g. Covarianza
h. Senderos (path) 
i. Log-lineal

j. Sistemas

k. Otro

9. ¿Qué tipo de análisis multivariable exploratorio se realiza?
a. Clústers
b. Correspondencias
c. Estructura latente
d. Escalonamiento multidimensional
e. Otro

10. ¿Cuál es el tipo de análisis secundario que se realiza?
a. Cualitativo
b. Cuantitativo
c. No consta

11. ¿Qué tipo de análisis estadístico o matemático se realiza en función del número de variables?
a. Univariable
b. Bivariable
c. Multivariable confirmatorio
d. Multivariable exploratorio

12. ¿Qué tipo de análisis cualitativo se realiza?
a. Inducción analítica
b. Teoría fundamentada
c. Semiótico-estructural

13. ¿Qué tipo de análisis univariable se realiza?

14. ¿Qué tipo de análisis bivariable se realiza?

15. ¿Qué tipo de análisis multivariable confirmatorio se realiza?
a. Componentes principales
b. Regresión múltiple
c. Correlaciones canónicas
d. Discriminante 

e. Factorial
f. Varianza
g. Covarianza
h. Senderos (path)
i. Log-lineal
j. Sistemas
k. Otro

16. ¿Qué tipo de análisis multivariable exploratorio se realiza?
a. Clústers
b. Correspondencias
c. Estructura latente
d. Escalonamiento multidimensional
e. Otro 
